\title{
Cooperative Game Approaches to Measuring Network Reliability Considering Paradoxes
}

\author{
W.Y. Szeto \\ Department of Civil Engineering, The University of Hong Kong, Hong Kong
}

\begin{abstract}
Traditionally, game-theoretic approaches to measuring transport network reliability have relied on the outcome of a game played between on the one hand users who seek minimum cost routes, and on the other hand, one or more evil entities or demons that seek to maximize the total expected network cost to the users by damaging links in the network. As the demons are assumed to be noncooperative, this approach has been criticized that it cannot produce the worst-case solution for reliability analysis, contradicting the original purpose of adopting game-theoretic approaches. In this paper, two cooperative game formulations, the Stackelberg-Nash formulation and the partial-cooperative Nash formulation, are proposed to determine travel cost reliability. Their relationships are analyzed and their properties are examined. This paper also investigates under what condition(s) the classical non-cooperative demon behavior can lead to the worst-case solution. Numerical studies are provided to demonstrate (i) the effects of the number of coalitions formed by demons on total network expected cost and network/OriginDestination (OD) travel cost reliability; (ii) the paradoxical phenomena that if one adds a road to a network then all the travelers may be worse off in terms of expected network travel cost and network travel cost reliability respectively, and (iii) the possibility of the classical game-theoretic approach of overestimating network/OD travel cost reliability.
\end{abstract}

\section{Introduction}

In the past decade, transport network reliability has received much attention. This has occurred for at least two reasons. First, the experience of events such as the Kobe earthquake of 1995 have led many researchers to identify the transport network as a critical element in acting as a lifeline to ferry emergency services to and from the scene of a major incident in the network. Second, increased economic activity worldwide has led to an increase in the values of time and requirement on the network performance, especially under disruptions like adverse weather, traffic accidents, breakdowns, signal failures, road-works, landslides, and terrorist attacks.

Many dimensions of transport network reliability have been addressed in the literature. They include but not limited to the following: 
- Connectivity reliability is defined as the probability that specific OD pairs in a network remain connected when links are subject to complete failures (Iida and Wakayabashi, 1989). Capacity constraints and travel time are not considered in this reliability. As such, this measure is most appropriate for the modeling of extreme events such as earthquakes.

- Capacity reliability is defined as the probability that the transportation system can accommodate a given demand level at an acceptable level of service, while taking the route choice behavior into account (Chen et a. 1999; 2002). This reliability can include connectivity reliability as a special case.

- Travel time reliability is normally concerned with the probability that a trip on a particular path can be made successfully within a given acceptable time threshold (Asakura and Kashiwadani, 1991). This concept has been generalized, refined, and extended to consider path, origindestination and network travel times (or cost).

- Travel demand satisfaction reliability is concerned with the probability that the ratio of the equilibrium to latent travel demand is not less than certain acceptable value (Heydecker et al., 2007). The travel demand satisfaction reliability can be extended to include other reliability measures such as travel time reliability under certain conditions.

- Behavior reliability considers the effect of travel time reliability in the route choice behavior of travelers. Very often, both the mean and variance of travel times are incorporated in an equilibrium framework to depict route choice behavior under travel time uncertainty. Some example works include Lo and Tung (2003), Lo et al. (2006), Lam et al. (2008), and Siu and Lo (2008).

Among them, travel time reliability or its extension, travel cost reliability, received most attention in the past (e.g., Asakura and Kashiwadani, 1991; Taylor, 1999; Yang et al., 2000; Chen et al., 2002, 2007; Lo, 2002; Al-Deek and Emam, 2006; Nie and Fan, 2006; Sumalee et al., 2006; Kaparias et al., 2008; Shao et al., 2008; Sumalee and Watling, 2008; Higatani et al., 2009).

The travel time/cost reliability can be studied through the game-theoretic approach (e.g., Bell, 2000; Bell and Cassir, 2002; Szeto et al., 2006, 2007) which does not require statistical distributions for link performance (such as delay, travel time or capacity). This approach is very attractive especially when the distributions are absent or inaccurate. In addition, the game-theoretic approach can determine links where the risk-averse network users are the most vulnerable to link capacity degradation. The approach can also provide normative measures of network reliability for pointing out the most vulnerable/critical nodes, paths, and OD pairs and comparison of different designs as mentioned by Bell (2000).

The main idea behind the game-theoretic approach is based on the notion of a fictitious game played between on the one hand users who seek minimum cost routes, and on the other hand, one or more evil entities or demons that seek to maximize the total expected network cost to the users by damaging links in the network. Traditionally, this game consists of two sub-problems: the user problem 
and the demon problem. The user problem describes the non-cooperative behavior of network users, whereas the demon problem describes the evil behavior in the sense of trying to cause maximum damage to the users. These two problems are solved simultaneously to obtain solutions and/or reliabilities. One may criticize that this classical approach can only give a non-cooperative Nash equilibrium solution and cannot give the worst-case network reliability and most vulnerable links and nodes, especially when there is more than one demon. The demons should work together to maximize the damage so that the worst-case scenario can be analyzed and the worst-case performance measures like total network cost and travel time reliability can be determined. Moreover, like the traditional user-equilibrium assignment, the users are non-cooperative. One may ask whether some paradoxical phenomena similar to Braess' paradox (Braess, 1968) can be observed.

To address the criticism and query, this paper proposes two game-theoretic formulations to measuring network reliability, namely the Stackelberg-Nash formulation and the partial-cooperative Nash formulation. Like classical gametheoretic formulations for measuring network reliability, each of the two formulations consists of two problems: the demon problem and the user problem. For the Stackelberg-Nash formulation, the demon problem is the upper level problem. In this problem, the demons are assumed to be cooperative to maximize the impact to the users by taking their responses into account but have no direct control on their route choice. The overall damage strategies are assumed to be coordinated by one demon coordinator. The user problem is the lower level problem which describes the risk-averse route selection behavior of the users, given the damage information from the upper level problem. For the partial cooperative-Nash formulation, the demon problem assumes that there is one coordinator to coordinate the demons' link selection strategies to maximize the impact to the users but does not take their reaction into account. The user problem is identical to that of the classical gametheoretic formulations but assumes that all the users play a non-cooperative Nash game with the coordinator.

This paper examines the properties of the two game-theoretic formulations, and discusses the relationships between the two proposed formulations and the multidemon formulation proposed in Szeto et al. (2007). In particular, this paper investigates under what condition(s), the multi-demon formulation and the two proposed formulations will give the same worst-case result. This paper also conducts a brief study to demonstrate the existence of two analogues of Braess' paradox for expected network travel cost and network travel cost reliability in both the classical and proposed game-theoretic approaches, namely stochastic Braess' paradox and travel cost reliability paradox, that if one adds a road to a network then all the travelers may be worse off in terms of expected network travel cost and network travel cost reliability respectively. Numerical studies are also performed to illustrate the effects of the number of coalitions formed by demons on total network expected cost and network/Origin-Destination (OD) travel cost reliability, and the possibility of the classical approach of overestimating network/OD travel cost reliability. 
The remainder of this paper is organized as follows: Section 2 proposes and analyzes the Stackelberg-Nash formulation. Section 3 discusses the proposed partial-cooperative Nash formulation and its relationship with the Stackelberg-Nash formulation. Section 4 examines some properties of the multi-demon formulation and its relationship with the two proposed formulations. Section 5 defines the mathematical conditions of the two paradoxes. Section 6 details the numerical studies. Finally, section 7 provides some concluding remarks.

\section{The Stackelberg-Nash Formulation}

Consider a general transportation network with multiple OD pairs, $N^{r s}$ homogenous users between each OD pair $r s$ and $M$ homogenous demons. The $N^{r s}$ homogenous users are assumed to seek their minimum expected cost routes in a non-cooperative manner. The $M$ homogenous demons have the freedom to roam the network without restriction and the capability to select any link to damage, including links already selected for damage by other demon(s). Their intention is to maximize the expected travel costs of the users by damaging link(s) in the network. However, unlike Szeto et al. (2007), these $M$ demons are allowed to be cooperative with each other when selecting links for damage so that every demon will be better off by forming coalitions. Moreover, when they cooperatively select a set of links to damage, they are assumed to take into account of the reaction of the users and create the worst-case scenario for the users in terms of the total expected travel cost of all users (i.e., total expected network cost). With this setting, the first proposed problem can be expressed as a bi-level problem as described below.

\subsection{The Upper Level Problem: The Cooperative Demon Problem}

The upper level problem is the demon problem, where all demons aim to maximize their individual payoff by selecting links for damage. Their payoffs are all equal to the total expected network cost TENC divided by the number of demons $M$. They are also free to form coalitions to cooperatively select links for damage without losing any payoff. As more cooperation between demons can result in higher individual payoff in general (as shown in the numerical study later), the natural outcome for all $M$ demons is to form one coalition so that all demons can cooperatively select links to damage, thereby maximizing all demons' payoffs simultaneously. When all demons' payoffs are maximized, the total payoff to all demons must also be maximized. Hence, the demon problem can be simplified as the one where there is one demon coordinator who selects a mixed strategy over all possible scenarios (defined as the combinations of links selected by all $M$ de- 
mons) to maximize the total payoff $(T P)$ to all the demons taking the user reaction into account.

The total payoff can be mathematically formulated as:

$T P=T E N C=\sum_{a=1}^{n} \sum_{k=1}^{K} v_{a}^{*} t_{a k}\left(v_{a}^{*}\right) q_{k}$,

where $v_{a}{ }^{*}$ is the optimal flow on link $a$ taking the user reaction into account; $t_{a k}($.$) denotes the travel cost on link a$ in scenario $k ; q_{k}$ is the probability of scenario $k ; n$ and $K$ denote the numbers of links in the network and scenarios respectively.

By definition, the scenario probability $q_{k}$ in (1) must satisfy:

$\sum_{k} q_{k}-1=0$, and

$q_{k} \geq 0, \forall k$

\subsection{The Lower-Level Problem: The Non-Cooperative User Problem}

The user problem can be viewed as a non-cooperative game in which each homogenous player tries to select the route with minimum expected cost given $\mathbf{q}=\left[q_{k}\right]$. This user problem can be approximated to deterministic user equilibrium assignment when the number of homogenous users is large (see Bell and Cassir, 2002). The formulation of this problem is as follows:

$\min _{\mathbf{h}} Z=\sum_{a} \sum_{k} q_{k} \int_{0}^{v_{a}(\mathbf{h})} t_{a k}(x) d x$

subject to

$v_{a}=\sum_{r s} \sum_{j} \delta_{j a} h_{j}^{r s}, \forall a$,

$N^{r s}=\sum_{j} \delta_{j}^{r s} h_{j}^{r s}, \forall r s$, 
$h_{j}^{r s} \geq 0, \forall r s, j$,

where $\mathbf{h}$ is the route flow vector; $v_{a}$ is the flows on link $a ; h_{j}^{r s}$ is the flows on route $j$ between OD pair $r s ; \delta_{j a}$ is the route-link incidence indicator which is 1 if link $a$ is on path $j$, and 0 otherwise; $\delta_{j}^{r s}$ is the route-OD incidence indicator. $\delta_{j}^{r s}=1$ if $j$ connects OD pair $r s$, and $\delta_{j}^{r s}=0$ otherwise.

Equation (5) depicts the relationship between route and link flows. Equation (6) states that the demand of an OD pair is equal to sum of the flows on the paths connecting the OD pair. Condition (7) is the non-negativity constraint.

Let $O(z)$ be the set of outbound links emanating from node $z$ and $I(z)$ be the set of inbound links feeding into node $z$. Equations (5) and (6) can then be totally replaced by the flow conservation equations at all nodes (except the origin nodes as those equations are redundant):

$\sum_{a \in O(z)} v_{a}-\sum_{b \in I(z)} v_{b}-Q_{z}=0, \forall z$,

where $Q_{z}=\left\{\begin{array}{ll}-\sum_{s} N^{r s}, & \text { if } z=s ; \\ 0, & \text { otherwise. }\end{array}\right.$ Moreover, (7) is replaced by

$v_{a} \geq 0, \forall a$.

Hence, the problem (4)-(7) can be rewritten based on link flows as decision variables.

The existence and uniqueness of solutions to the lower level problem depends on the choice of link cost functions.

Proposition 1: For a given $\mathbf{q}=\left[q_{k}\right]$ from the upper level problem, the lower level problem (4), (8) and (9) has at least one optimal link flow pattern if the link travel cost is continuous with respect to its link flows.

Proof: As the travel cost function is a continuous function of its link flows, the definite integral and their linear combinations are also continuous with respect to link flows. Hence, the objective function is a continuous function of link flows. Moreover, the solution set (8) - (9) is non-empty and compact. By Weierstrass' Theorem, a solution exists to the lower level problem (4), (8) and (9) .

At least two link cost functions can lead to the existence of solutions. The first one is the separable and continuously differentiable function used in Bell and Cassir (2002) and Szeto et al. (2006, 2007): 
$t_{a k}\left(v_{a}\right)=\alpha_{a}+\beta_{a}\left(\frac{v_{a}}{c_{a k}}\right)^{\gamma_{a}}$,

where $\alpha_{a}>0$ is the free-flow travel cost of link $a ; \beta_{a}>0$ and $\gamma_{a}>1$ are the delay parameter for link $a$ respectively; $c_{a k}$ is the capacity of link $a$ in scenario $k$, which equals the normal capacity when no demon selects this link for damage in this scenario, and equals reduced capacity otherwise. The reduced capacity depends on the assumptions adopted. The two common assumptions used in Szeto et al. (2007) are as follows:

1. The reduced capacity is always $50 \%$ of the normal capacity regardless of the number of demons selecting the link for damage, and

2. The capacity is either linear or nonlinear decreasing with the number of demons selecting the link for damage.

The second link travel cost function is:

$t_{a k}\left(v_{a}\right)=\frac{\beta_{a k}}{c_{a k}}$,

which is independent of link flows. This one is the generalization of the link cost function in Bell (2000).

Proposition 2: If the link cost function is separable, increasing, and differentiable with respect to its link flow, the lower level problem (4), (8) and (9) has only one link flow solution for each given $\mathbf{q}=\left[q_{k}\right]$.

Proof: Under the assumption, $\frac{d t_{a k}\left(v_{a}\right)}{d v_{b}}=0, a \neq b$ and $\frac{d t_{a k}\left(v_{a}\right)}{d v_{a}}>0$. Moreover, by the definition of probability, there is at least one $q_{k}>0$. Therefore, $\frac{\partial Z^{2}}{\partial v_{a} v_{b}}=\left\{\begin{array}{cl}\frac{d\left(\sum_{k} t_{a k} q_{k}\right)}{d v_{a}}>0 & \text { for } a=b ; \text { Hence, the Hessian of } Z \text { is positive defi- } \\ 0 & \text { otherwise. }\end{array}\right.$ nite and the objective function $Z$ is strictly convex.

As the feasible solution set formed by linear constraints is convex, and the union of the positive orthant and a convex set is also convex, the feasible region is convex.

Since the problem has a convex objective function and a convex solution set, the problem has a unique link flow solution.

Proposition 2 implies that when the link cost function (10) is adopted, the lower level problem has a unique link flow solution. However, like standard user equi- 
librium, the route flow solutions are not unique in general, as the Hessian of $Z$ with respect to path flows may not be positive definite.

The optimality conditions of the problem (4)-(7) are the risk-averse traffic assignment conditions as in Szeto et al. (2007):

$$
\begin{aligned}
& h_{j}^{r s}\left[\sum_{k} g_{j k}^{r s}(\mathbf{h}) q_{k}-g_{\text {min }}^{r s}\right]=0, \forall r s, j, \\
& \sum_{k} g_{j k}^{r s}(\mathbf{h}) q_{k}-g_{\min }^{r s} \geq 0, \forall r s, j,
\end{aligned}
$$

where $g_{\min }^{r s}$ in (12) and (13) is the Lagrangian multiplier associated with (6); $g_{j k}^{r s}(\mathbf{h})$ represents the travel cost of route $j$ between OD pair $r s$ in scenario $k$ based on the flow vector $\mathbf{h}$ and equals the sum of the travel cost on every link on this route:

$g_{j k}^{r s}(\mathbf{h})=\sum_{a} \delta_{j}^{r s} \delta_{j a} t_{a k}\left(v_{a}\right), \forall r s, j$

According to (12), if route $j$ connecting OD pair $r s$ carries flow $\left(h_{j}^{r s}>0\right)$, the corresponding expected route travel cost $\sum_{k} g_{j k}^{r s}(\mathbf{h}) q_{k}$ must be equal to the Lagrangian multiplier $g_{\min }^{r s}$, as the term inside the square brackets in (12) must equal zero. If route $j$ carries no flow $\left(h_{j}^{r s}=0\right.$ ), the corresponding expected route travel cost must be greater than or equal to the Lagrangian multiplier based on (13). Hence, the Lagrangian multiplier can be interpreted as the minimum expected travel cost between OD pair $r s$. That is, $g_{\text {min }}^{r s}=\min _{\mathrm{d}}\left(\sum_{k} g_{d k}^{r s}(\mathbf{h}) q_{k}\right)$, where $d$ is the minimum expected travel cost route between OD pair $r s$. Since free flow travel cost is positive, the Lagrangian multiplier must be greater than zero:

$g_{\min }^{r s}>0$

\subsection{The Stackelberg-Nash Problem}

The Stackelberg-Nash problem can be expressed as follows: 


$$
\max _{\mathbf{q}} T P=\sum_{a=1}^{n} \sum_{k=1}^{K} v_{a}^{*} t_{a k}\left(v_{a}^{*}\right) q_{k}
$$

subject to (2)-(3), where $v_{a}^{*}$ is the optimal vector obtained from

$$
\underset{\mathbf{v}}{\operatorname{Min}} Z=\sum_{a} \sum_{k} q_{k} \int_{0}^{v_{a}} t_{a k}(x) d x
$$

subject to (8)-(9), where $\mathbf{v}=\left[v_{a}\right]$ is the link flow vector. The lower level problem can also be replaced by

$$
\min _{\mathbf{h}} Z=\sum_{a} \sum_{k} q_{k} \int_{0}^{v_{a}(\mathbf{h})} t_{a k}(x) d x
$$

subject to (5)-(7).

These bilevel problems must have a solution and the solution is well-defined, because

1. the lower level problem has a unique $\mathbf{v}^{*}$ for each given $\mathbf{q}$ (proposition 2);

2. the feasible solution set for the upper level problem is non-empty, convex and bounded, and;

3. the objective function in the upper level problem is continuous.

The Stackelberg-Nash problem can also be expressed as:

$$
\max _{\mathbf{q}, \mathbf{h}, \mathbf{g}_{\min }} T P=\sum_{a=1}^{n} \sum_{k=1}^{K} v_{a} t_{a k}\left(v_{a}\right) q_{k}=\sum_{r s} \sum_{j} \sum_{k=1}^{K} h_{j}^{r s} g_{j k}^{r s}(\mathbf{h}) q_{k}
$$

subject to (2)-(3), (5)-(7), (12)-(15), where $\mathbf{g}_{\min }=\left[\begin{array}{c}g_{\min }^{r s} \\ \end{array}\right.$.

The first-order conditions of the Stackelberg-Nash problem include constraints (2)-(3), (6)-(7), (12)-(15), and the following:

$$
\begin{aligned}
& q_{k}\left[\pi_{q}-\sum_{r s} \sum_{j} h_{j}^{r s} g_{j k}^{r s}(\mathbf{h})\right]=0, \forall k, \\
& \pi_{q}-\sum_{r s} \sum_{j} h_{j}^{r s} g_{j k}^{r s}(\mathbf{h}) \geq 0, \forall k, \\
& h_{j}^{r s}\left[\sum_{k} q_{k}\left[g_{j k}^{r s}(\mathbf{h})\right]+\sum_{k} \sum_{e f} \sum_{l} h_{l}^{e f} \frac{\partial g_{l k}^{e f}(\mathbf{h})}{\partial h_{j}^{r s}} q_{k}-\mu^{r s}\right]=0, \forall r s, j,
\end{aligned}
$$


$\sum_{k} q_{k}\left[g_{j k}^{r s}(\mathbf{h})\right]+\sum_{k} \sum_{e f} \sum_{l} h_{l}^{e f} \frac{\partial g_{l k}^{e f}(\mathbf{h})}{\partial h_{j}^{r s}} q_{k}-$

$v_{j}^{r s}\left[\sum_{k} g_{j k}^{r s}(\mathbf{h}) q_{k}-g_{\min }^{r s}\right]-\mu^{r s} \geq 0, \forall r s, j$,

$u_{j}^{r s}\left[\sum_{k} g_{j k}^{r s}(\mathbf{h}) q_{k}-g_{\text {min }}^{r s}\right]=0, \forall r s, j$

$\sum_{j}\left(v_{j}^{r s} h_{j}^{r s}+u_{j}^{r s}\right)=0, \forall r s$, and

$u_{j}^{r s} \leq 0, \forall r s, j$,

where $\pi_{q}, \mu^{r s}, v_{j}^{r s}$, and $u_{j}^{r s}$ are the Lagrangian multipliers of (2), (6), (12), and (13), respectively.

In (16) and (17), $\sum_{r s} \sum_{j} h_{j}^{r s} g_{j k}^{r s}(\mathbf{h})$ is the total expected network cost in scenario $k$ or the total payoff of all the demons when the coordinator selects coordination strategy $k$. This total expected cost is obtained by $\frac{\partial T P}{\partial q_{k}}$. The multiplier $\pi_{q}$ in (16) and (17) can be interpreted as the maximum total payoff. Then, according to equation (16), when the coordinator selects coordination strategy $k$, the corresponding total payoff must be equal to the maximum payoff $\pi_{q}$. Condition (17) states that any coordinator's strategy cannot produce the total payoff larger than the maximum total payoff.

In (18) and (19), $\sum_{k} q_{k}\left[g_{j k}^{r s}(\mathbf{h})\right]+\sum_{k} \sum_{e f} \sum_{l} h_{l}^{e f} \frac{\partial g_{l k}^{e f}(\mathbf{h})}{\partial h_{j}^{r s}} q_{k}$ is the marginal expected path cost with respect to route flows $h_{j}^{r s}$ (i.e., $\frac{\partial T P}{\partial h_{j}^{r s}}$ ). In particular, equation (18) states that the marginal expected path costs on all used routes must be equal at optimality. Condition (19) implies that the marginal expected path cost on any unused route can be greater than, equal to or smaller than that on the used route. 
Condition (20) has a very similar form with (12), which implies that $h_{j}^{r s}=0$ if and only if $u_{j}^{r s}=0$, and that $h_{j}^{r s}$ and $u_{j}^{r s}$ have opposite signs. Equation (21) describes the relationships between $h_{j}^{r s}, u_{j}^{r s}$ and $v_{j}^{r s}$. Constraint (22) is the sign restriction for $u_{j}^{r s}$.

\section{The Partial-cooperative Nash Formulation}

The second proposed problem, namely the partial-cooperative Nash problem, is similar to the Stackelberg-Nash problem except that the coordinator does not take the user reaction into account. The partial-cooperative problem is to find $\mathbf{q}$ to maximize $T P$ and $\mathbf{h}$ to minimize $Z$ simultaneously subject to (2)-(3), and (5)-(7). The first-order conditions include (2)-(3), (5)-(7), and (12)-(17).

Proposition 3: When all link travel costs are flow-independent, the Stackelberg-Nash solution is identical to the solution of the Nash game played between the coordinator and the whole user group, and the solution of the Nash game played between the coordinator and all the users.

Proof: When link costs are flow independent, $Z=\sum_{a} \sum_{k} q_{k} \int_{0}^{v_{a}} t_{a k}(x) d x=\sum_{a} \sum_{k} q_{k} t_{a k} v_{a}=T P$. The Stackelberg-Nash formulation can be rewritten as the following Max-Min formulation:

$\underset{\mathbf{q}}{\operatorname{Max}} \operatorname{Min}_{\mathbf{h}} T P$

subject to (2)-(3), and (5)-(7).

By the minimax theorem, $\underset{\mathbf{q}}{\operatorname{Max}} \operatorname{Min}_{\mathbf{h}} T P=\underset{\mathbf{h}}{\operatorname{Min}} \underset{\mathbf{q}}{\operatorname{Max}} T P$. Then, the following Nash equilibrium conditions between the coordinator and the whole user group are obtained:

$$
\begin{aligned}
& T P\left(\mathbf{h}^{*}, \mathbf{q}^{*}\right)=\max _{\mathbf{q}} \min _{\mathbf{h}} T P \geq T P\left(\mathbf{h}^{*}, \mathbf{q}\right) \text { and } \\
& T P\left(\mathbf{h}^{*}, \mathbf{q}^{*}\right)=\min _{\mathbf{h}} \max _{\mathbf{q}} T P \leq T P\left(\mathbf{h}, \mathbf{q}^{*}\right),
\end{aligned}
$$

where $\operatorname{TP}(\mathbf{h}, \mathbf{q})$ is the payoff at $(\mathbf{h}, \mathbf{q})$. As all the users are non-cooperative with each other, all the users and the coordinator are non-cooperative with each other in this game.

Note that when link cost functions are flow-dependent, an optimal solution to the partial-cooperative Nash problem may not be optimal to the Stackelberg-Nash problem as the solution may not satisfy (18)-(22). However, this optimal solution is feasible to the Stackelberg-Nash problem as stated below: 
Proposition 4: Any optimal solution to the partial-cooperative Nash problem is a feasible solution to the Stackelberg-Nash problem.

Proof: As any optimal solution of the partial-cooperative Nash problem must satisfy (2)-(3), (6)-(7), and (12)-(17), the optimal solution must be feasible to the Stackelberg-Nash problem which has fewer constraints: (2)-(3), (6)-(7), and (12)(15).

\section{The Multi-demon Formulation}

For completeness, the multi-demon problem in Szeto et al. (2007) is briefly reviewed here. The multi-demon problem consists of two subproblems: the user problem and the demon problem. The user problem is described by the first-order conditions (5)-(7) and (12)-(14) where the scenario probability $q_{k}$ and $g_{\min }^{r s}$ in (12)-(14) are respectively defined as follows:

$q_{k}=\prod_{m=1}^{M} p_{l_{m}}^{m}$, and

$g_{\min }^{r s}=\min _{\mathrm{d}}\left(\sum_{k} g_{d k}^{r s}(\mathbf{h}) q_{k}\right)$,

where $p_{l_{m}}^{m}$ is the probability of demon $m$ selecting link $l_{m}$ to damage.

The demon problem describes the objective of each non-cooperative demon. Each demon seeks its mixed strategy to maximize its expected pay-off $P$ :

$P=\sum_{l_{1}=1}^{n} \ldots \sum_{l_{M}=1}^{n}\left\{\left(\prod_{i=1}^{M} p_{l_{i}}^{i}\right)\left[\sum_{a} t_{a, l_{1} \ldots, l_{m}, \ldots, l_{M}}(\mathbf{v}) v_{a}\right]\right\}$,

where $t_{a, l_{1} \ldots, l_{m}, \ldots, l_{M}}(\mathbf{v})$ denotes the flow-dependent cost on link $a$ in scenario $k=\left(l_{1}, \ldots, l_{m}, \ldots, l_{M}\right)$ with demon $m$ selecting link $l_{m}$ for damage.

Let

$\theta_{l_{\mathrm{m}}}^{m}=\frac{\partial P}{\partial p_{l_{m}}^{m}}=\prod_{i=1, i \neq m}^{M} p_{l_{i}}^{i}$ 
be the expected payoff to demon $m$ (or the total expected network cost to the users) when demon $m$ selects link $l_{m}$ to damage and considers the link selection strategies of all other demons. Let also

$\pi^{m}=\max _{l_{\mathrm{m}}} \theta_{l_{\mathrm{m}}}^{m}$.

The demon problem can then be described by the following conditions:

$p_{l_{m}}^{m}\left\{\pi^{m}-\theta_{l_{m}}^{m}\right\}=0, m=1, \ldots, M, l_{m}=1, \ldots, n$,

$\pi^{m}-\theta_{l_{m}}^{m} \geq 0, m=1, \ldots, M, l_{m}=1, \ldots, n$,

$p_{l_{m}}^{m} \geq 0, m=1, \ldots, M, l_{m}=1, \ldots, n$, and

$\sum_{l_{m}} p_{l_{m}}^{m}-1=0, m=1, \ldots, M$

The multi-demon problem is to find the link selection probability vector $\mathbf{p}=\left[p_{l_{m}}^{m}\right]$ and the route flow vector $\mathbf{h}$ to satisfy (5)-(7), (12)-(14), and (23)-(31) simultaneously. The solution is actually a Nash equilibrium solution. Three properties of this problem that have not been mentioned in the literature are given below:

Proposition 5: All the demons in the multi-demon problem must have the same expected payoff at optimality.

Proof: From (25), we can observe that all the demons receive the same expected payoff. Therefore, their maximum expected payoffs, which are the expected payoff evaluated at Nash equilibrium, must be the same. $\square$

Proposition 6: The set of optimal link selection probabilities of the multi-demon problem (5)-(7), (12)-(14) and (23)-(31) is identical to that of the revised multidemon problem with the payoff function $\mu P$, where $\mu>0$.

Proof: The revised formulation consists of (5)-(7), (12)-(14), (23), (24), (26), (27), (30), (31), and the following:

$P=\mu \sum_{l_{1}=1}^{n} \ldots \sum_{l_{M}=1}^{n}\left\{\left(\prod_{i=1}^{M} p_{l_{i}}^{i}\right)\left[\sum_{a} t_{a, l_{1} \ldots, l_{m}, \ldots, l_{M}}(\mathbf{v}) v_{a}\right]\right\}$, 
$p_{l_{m}}^{m}\left\{\mu \pi^{m}-\mu \theta_{l_{m}}^{m}\right\}=0, m=1, \ldots, M, l_{m}=1, \ldots, n$, and

$\mu \pi^{m}-\mu \theta_{l_{m}}^{m} \geq 0, m=1, \ldots, M, l_{m}=1, \ldots, n$.

As $\mu>0$, (33) and (34) can be respectively rewritten as:

$p_{l_{m}}^{m}\left\{\pi^{m}-\theta_{l_{m}}^{m}\right\}=0, m=1, \ldots, M, l_{m}=1, \ldots, n$, and

$\pi^{m}-\theta_{l_{m}}^{m} \geq 0, m=1, \ldots, M, l_{m}=1, \ldots, n$.

The formulation (5)-(7), (12)-(14), (23), (24), (26), (27), (30)-(32), (35) and (36) is actually the same as the formulation (5)-(7), (12)-(14), and (23)-(31). Therefore the two formulations have the same set of link selection probabilities.

Proposition 7: Any optimal solution of the multi-demon formulation is a feasible solution to both the Stackelberg-Nash problem and the partial-cooperative Nash problem under definition (23).

Proof: As mentioned before, under (23), the three user problems are equivalent. Hence, we only need to show that the optimal link selection probability vector $\left[p_{l_{m}}^{m} *\right]$ satisfies the constraints (2) and (3) in both the Stackelberg-Nash and Partial-cooperative Nash problems. As $p_{l_{m}}^{m} * \geq 0, \forall l_{m}, m, q_{k}=\prod_{m=1}^{M} p_{l_{m}}^{m} * \geq 0$. That is, (3) is satisfied. Moreover, since $\quad \sum_{l_{m}} p_{l_{m}^{m}}^{m} *-1=0, \forall m$, $\sum_{k} q_{k}=\sum_{l_{1}=1}^{n} \ldots \sum_{l_{M}=1}^{n}\left(\prod_{m=1}^{M} p_{l_{m}}^{m} *\right)=\prod_{m=1}^{M}\left(\sum_{l_{m}=1}^{n} p_{l_{m}}^{m} *\right)=1$. Therefore, (2) is also satisfied.

When $\mu=1 / M$, the payoff function of each demon in the two problems is the same. However, the revised multi-demon problem may not give the maximal $T E N C$ as the optimal solution of the revised multi-demon formulation is a feasible solution to the Stackelberg-Nash problem according to proposition 7 but may not be an optimal solution to the Stackelberg-Nash problem.

Proposition 8: Assume $P$ is locally concave of $p_{l_{m}}^{m}$ at optimality. Then, at least one optimal solution to the multi-demon problem with the payoff function $P$ gives an optimal solution to the partial-cooperative Nash problem. 
Proof: As $T P$ is a linear function of $q_{k}, T P$ must be concave at optimality and the necessary optimality conditions for the partial-cooperative Nash problem are also the sufficient optimality conditions. Moreover, under the given assumption, the necessary optimality conditions for the multi-demon problem are also the sufficient optimality conditions. Therefore, we only need to show that the first-order conditions of the partial-cooperative Nash problem imply those of the multidemon problem. By comparing the two sets of sufficient optimality conditions, we found that most of them are the same. Therefore, we only need to show that (16)(17) imply (28)-(29).

(17) $\Rightarrow$ (29):

By chain rule, $\frac{\partial T P}{\partial p_{l_{m}}^{m}}=\sum_{k^{\prime}} \frac{\partial T P}{\partial q_{k^{\prime}}} \frac{\partial q_{k^{\prime}}}{\partial p_{l_{m}}^{m}}$. As $T P=P$,

$\frac{\partial P}{\partial p_{l_{m}}^{m}}=\sum_{k^{\prime}} \frac{\partial T P}{\partial q_{k^{\prime}}} \frac{\partial q_{k^{\prime}}}{\partial p_{l_{m}}^{m}}$

From (17), $\sum_{r s} \sum_{j \in P^{r s}} h_{j}^{r s} g_{j k}^{r s}(\mathbf{h})=\frac{\partial T P}{\partial q_{k}} \leq \pi_{q}$. Hence,

$\frac{\partial P}{\partial p_{l_{m}}^{m}} \leq \sum_{k^{\prime}} \pi_{q} \frac{\partial q_{k^{\prime}}}{\partial p_{l_{m}}^{m}}=\pi_{q} \sum_{k^{\prime}} \frac{\partial q_{k^{\prime}}}{\partial p_{l_{m}}^{m}}$

From (23), $\frac{\partial q_{k}}{\partial p_{l_{m}}^{m}}=\sum_{l_{1}} \ldots \sum_{l_{i}, i \neq m} \ldots \sum_{l_{M}}\left(\prod_{i=1, i \neq m}^{M} p_{l_{i}}^{i}\right)$ where $k=\left(l_{1}, \ldots, l_{m}, \ldots, l_{M}\right)$. Hence (38) becomes $\frac{\partial P}{\partial p_{l_{m}}^{m}} \leq \pi_{q}\left[\sum_{l_{1}} \ldots \sum_{l_{i}, i \neq m} \ldots \sum_{l_{M}}\left(\prod_{i=1, i \neq m}^{M} p_{l_{i}}^{i}\right)\right]$, which be rewritten as

$\frac{\partial P}{\partial p_{l_{m}}^{m}} \leq \pi_{q} \prod_{i=1, i \neq m}^{M}\left(\sum_{l_{i}} p_{l_{i}}^{i}\right)$

Substituting (31) into (39) gets

$\frac{\partial P}{\partial p_{l_{m}}^{m}} \leq \pi_{q}$ 
According to (26) and (27), $\pi^{m}=\max _{l_{m}} \frac{\partial P}{\partial p_{l_{m}}^{m}}$. Because of (40),

$\pi^{m}=\max _{l_{m}} \frac{\partial P}{\partial p_{l_{m}}^{m}}=\pi_{q}$

Substituting (41) into (40) gets

$\frac{\partial P}{\partial p_{l_{m}}^{m}} \leq \pi^{m}$

Hence, $\frac{\partial T P}{\partial q_{k}} \leq \pi_{q}$ implies $\frac{\partial P}{\partial p_{l_{m}}^{m}} \leq \pi^{m}$.

(16) $\Rightarrow(28)$ :

Without loss of generality, the link selection probabilities can be classified into two groups, zero and positive. The link selection probabilities are positive if they constitute at least one scenario with a positive probability, and zero otherwise. Let $p_{l_{m}}^{m+}$ be the positive probability of demon $m$ selecting link $l_{m}$ to damage. Then, by definition of probability,

$\sum_{l_{m}} p_{l_{m}}^{m+}=1, \forall m$

Then, consider mutually exclusive two cases of the probability of scenario $k=\left(l_{1}, \ldots, l_{m}, \ldots, l_{M}\right): q_{k}>0$ and $q_{k}=0$. If $q_{k}>0$, all the link selection probabilities that constitute $q_{k}=\prod_{m=1}^{M} p_{l_{m}}^{m}$, must be positive. That is,

$q_{k}=\prod_{m=1}^{M} p_{l_{m}}^{m}>0 \Rightarrow p_{l_{m}}^{m}>0, \forall m$

Moreover, if $q_{k}>0$ and $p_{l_{m}}^{m}>0$,

$\frac{\partial q_{k}}{\partial p_{l_{m}}^{m}}=\prod_{i \neq m} p_{l_{i}}^{i}>0, \forall m$

Furthermore, by (16), 
if $q_{k}>0, \pi_{q}=\sum_{r s} \sum_{j} h_{j}^{r s} g_{j k}^{r s}(\mathbf{h})=\frac{\partial T P}{\partial q_{k}}$.

(45) and (46) imply

$\frac{\partial T P}{\partial q_{k}}=\pi_{q}$ when $\frac{\partial q_{k}}{\partial p_{l_{m}}^{m}}>0, \quad p_{l_{m}}^{m}>0, \forall m$ and $q_{k}>0$.

If $q_{k}=0$, some link selection probabilities that constitute $q_{k}$ is zero. In particular, if $q_{k}=0$ but $p_{l_{m}}^{m}>0$,

$q_{k}=\prod_{i} p_{l_{i}}^{i}=p_{l_{m}}^{m} \prod_{i \neq m} p_{l_{i}}^{i}=p_{l_{m}}^{m} \frac{\partial q_{k}}{\partial p_{l_{m}}^{m}}=0 \Rightarrow \frac{\partial q_{k}}{\partial p_{l_{m}}^{m}}=0$.

In addition, from (16) and (17),

if $q_{k}=0, \frac{\partial T P}{\partial q_{k}} \leq \pi_{q}$.

(48) and (49) imply

$\frac{\partial T P}{\partial q_{k}} \leq \pi_{q}$ when $\frac{\partial q_{k}}{\partial p_{l_{m}}^{m}}=0, p_{l_{m}^{m}}^{m}>0, \forall m$ and $q_{k}=0$.

Let $K^{+}$and $K^{0}$ be the sets of scenarios that have positive and zero probabilities respectively given that $p_{l_{m}}^{m}>0$. Then, from (37), we have

$\frac{\partial P}{\partial p_{l_{m}}^{m}}=\sum_{k^{\prime}} \frac{\partial T P}{\partial q_{k^{\prime}}} \frac{\partial q_{k^{\prime}}}{\partial p_{l_{m}}^{m}}=\sum_{k^{\prime} \in K^{+}} \frac{\partial T P}{\partial q_{k^{\prime}}} \frac{\partial q_{k^{\prime}}}{\partial p_{l_{m}}^{m}}+\sum_{k^{\prime} \in K^{0}} \frac{\partial T P}{\partial q_{k^{\prime}}} \frac{\partial q_{k^{\prime}}}{\partial p_{l_{m}}^{m}}$.

If $q_{k}>0$ and $p_{l_{m}}^{m}>0$, (51) can be simplified via conditions (47) and (50) as $\frac{\partial P}{\partial p_{l_{m}}^{m}}=\sum_{k^{\prime} \in K^{+}} \pi_{q} \frac{\partial q_{k^{\prime}}}{\partial p_{l_{m}}^{m}}$, which can be further simplified as

$\frac{\partial P}{\partial p_{l_{m}}^{m}}=\pi_{q} \sum_{k^{\prime} \in K^{+}} \frac{\partial q_{k^{\prime}}}{\partial p_{l_{m}}^{m}}=\pi_{q}\left[\sum_{l_{1}} \ldots \sum_{l_{i}, i \neq m} \ldots \sum_{l_{M}}\left(\prod_{i=1, i \neq m}^{M} p_{l_{i}}^{i+}\right)\right]=\pi_{q} \prod_{i=1, i \neq m}^{M}\left(\sum_{l_{i}} p_{l_{i}}^{i+}\right)$. 
Substituting (43) and (41) into (52) gives

$$
\frac{\partial P}{\partial p_{l_{m}}^{m}}=\pi^{m}
$$

As $q_{k}>0$ implies both $p_{l_{m}^{m}}^{m}>0, \forall m$ (from (44)) and $\frac{\partial P}{\partial p_{l_{m}}^{m}}=\pi^{m}$ (from (53)), we have $\frac{\partial P}{\partial p_{l_{m}}^{m}}=\pi^{m}$ when $p_{l_{m}}^{m}>0, \forall m$, which is the alternative expression of (28).

The above proposition implies that the partial-cooperative Nash problem seeks the optimal Nash solution that yields the maximum TENC. In addition, if an optimal Nash equilibrium solution is also an optimal partial-cooperative Nash solution and satisfies conditions (18)-(22), then the Nash solution is also an optimal Stackelberg-Nash solution, and the Stackelberg-Nash problem can be viewed as seeking the optimal Nash solution that satisfies conditions (18)-(22) and yield the maximum TENC. One example in Section 6 illustrates this point. The following two propositions discuss two special cases.

Proposition 9: Assume $P$ is locally concave of $p_{l_{m}}^{m}$ at optimality and all the optimal solutions to the multi-demon problem give the same payoff. Then, all the optimal solutions to the multi-demon problem with the payoff function $P$ give an optimal solution to the partial-cooperative Nash problem.

Proof: This follows directly from Proposition 8.

Proposition 10: Assume $P$ is locally concave of $p_{l_{m}}^{m}$ at optimality and all the optimal solutions to the multi-demon problem give the same payoff and satisfy (18)-(22). Then, all the optimal solutions to the multi-demon problem with the payoff function $P$ give an optimal solution to the Stackelberg-Nash problem.

Proof: This follows from Proposition 8 and the fact that a Nash solution produces an optimal Stackelberg-Nash solution when the optimal Nash equilibrium solution is also an optimal partial-cooperative Nash solution and satisfies conditions (18)-(22). 


\section{The Stochastic Braess Paradox and Travel Cost Reliability Paradox}

Two analogues of Braess' paradox for total expected network cost and travel cost reliability in the proposed problems, namely stochastic Braess' paradox and reliability-paradox, are mathematically defined in this section.

The stochastic Braess paradox refers to the phenomena that if one adds a road to a network then all the travelers may be worse off in terms of total expected network cost. This paradox occurs when $T E N C_{\text {after }}-T E N C_{\text {before }}>0$, where $T E N C_{\text {before }}$ and $T E N C_{\text {after }}$ are respectively the total expected network costs before and after link addition.

The travel cost reliability paradox is the phenomena that if one adds a road to a network then all the travelers may be worse off in terms of network travel cost reliability. This paradox occurs when $R_{\text {after }}-R_{\text {before }}<0$, where $R_{\text {before }}$ and $R_{\text {after }}$ are the network travel cost reliability before and after link addition. The network travel cost reliability is defined as the probability of the total expected network cost to be less than the predefined tolerance.

\section{Numerical Studies}

\subsection{Stackelberg-Nash Solutions and Partial-cooperative Nash Solutions as Special Nash Solutions}

This example is set up to show the existence of multiple Nash solutions where some of them can be Stackelberg-Nash solutions and partial-cooperative Nash solutions. The network with one OD pair and three identical parallel links, links 1-3, is adopted for this purpose. We consider three demons, namely demons 1, 2 and 3. This leads to three cases: 1) one coalition (i.e., all demons cooperate with each other), 2) two coalitions (i.e., two demons cooperate against the third one) and 3) three coalitions (i.e., all demons are non-cooperative). In all cases, it is assumed that the capacity of each link is reduced by half if at least one demon selects that link for damage. The following parameters are adopted for illustrative purposes:

- $\quad N^{r s}=8000$;

- $\alpha_{1}=\alpha_{2}=\alpha_{3}=\$ 10$

- $\beta_{1}=\beta_{2}=\beta_{3}=\$ 1.5$;

- $\gamma_{1}=\gamma_{2}=\gamma_{3}=4$;

- $\quad c_{a k}= \begin{cases}4000 \mathrm{vph} & \text { if link } a \text { is selected by any demon; } \\ 2000 \mathrm{vph} & \text { otherwise. }\end{cases}$ 
The result for case 1 is obtained by solving the partial-cooperative Nash problem whereas the results for cases 2 and 3 are obtained by solving the multi-demon formulation. Note that for case 2 , we assume that there is a coordinator for determining the link selection strategies for the two demons and the coordinator is assumed to compete with the third demon.

When all demons are non-cooperative (case 3), the multi-demon formulation indeed gives seven Nash solutions as shown in Table 1. Solutions 2-7 can actually produce the partial-cooperative Nash solutions obtained in case 1, and give a probability of 1 for the scenario where the three demons select different links for damage. This means that some Nash solutions can also be the partial-cooperative Nash solutions. However, in terms of payoff, solutions 2-7 give the maximum payoff to each demon while solution 1 gives a suboptimal payoff to each demon. Not surprisingly, solutions 2-7 are also the solutions to case 2 and the payoff of each demon is maximal.

Table 1

Multiple link selection probabilities

a) Solution 1

\begin{tabular}{|l|l|l|l|}
\hline & Link 1 & Link 2 & Link 3 \\
\hline Demon 1 & $1 / 3$ & $1 / 3$ & $1 / 3$ \\
\hline Demon 2 & $1 / 3$ & $1 / 3$ & $1 / 3$ \\
\hline Demon 3 & $1 / 3$ & $1 / 3$ & $1 / 3$ \\
\hline
\end{tabular}

b) Solution 2

c) Solution 3

\begin{tabular}{|l|l|l|l|}
\hline & Link 1 & Link 2 & Link 3 \\
\hline Demon 1 & 0 & 0 & 1 \\
\hline Demon 2 & 1 & 0 & 0 \\
\hline Demon 3 & 0 & 1 & 0 \\
\hline
\end{tabular}

\begin{tabular}{|l|l|l|l|}
\hline & Link 1 & Link 2 & Link 3 \\
\hline Demon 1 & 1 & 0 & 0 \\
\hline Demon 2 & 0 & 1 & 0 \\
\hline Demon 3 & 0 & 0 & 1 \\
\hline
\end{tabular}

e) Solution 5

\begin{tabular}{|l|l|l|l|}
\hline & Link 1 & Link 2 & Link 3 \\
\hline Demon 1 & 0 & 0 & 1 \\
\hline Demon 2 & 0 & 1 & 0 \\
\hline Demon 3 & 1 & 0 & 0 \\
\hline
\end{tabular}

d) Solution 4
\begin{tabular}{|l|l|l|l|}
\hline & Link 1 & Link 2 & Link 3 \\
\hline Demon 1 & 0 & 1 & 0 \\
\hline Demon 2 & 1 & 0 & 0 \\
\hline Demon 3 & 0 & 0 & 1 \\
\hline
\end{tabular}

f) Solution 6

\begin{tabular}{|l|l|l|l|}
\hline & Link 1 & Link 2 & Link 3 \\
\hline Demon 1 & 0 & 1 & 0 \\
\hline Demon 2 & 0 & 0 & 1 \\
\hline Demon 3 & 1 & 0 & 0 \\
\hline
\end{tabular}

g) Solution 7

\begin{tabular}{|l|l|l|l|}
\hline & Link 1 & Link 2 & Link 3 \\
\hline Demon 1 & 1 & 0 & 0 \\
\hline Demon 2 & 0 & 0 & 1 \\
\hline Demon 3 & 0 & 1 & 0 \\
\hline
\end{tabular}

As the three parallel links are identical, all paths must carry equal flows and conditions (18)-(21) must be satisfied at optimality, including the equal marginal 
expected path cost condition. Hence, the partial-cooperative Nash solution is also a Stackelberg-Nash solution. This has been checked by solving the StackelbergNash problem directly via an existing global optimization software package.

To sum up, this example shows that Stackelberg-Nash solutions and partial cooperative Nash solutions can be obtained via the non-cooperative Nash formulation like the multi-demon formulation. However, when there is more than one Nash solution, there is no guarantee that Stackelberg-Nash solutions and partialcooperative Nash solutions can be obtained by solving the multi-demon formulation once.

\subsection{The Effect of Demon Coalitions to Total Expected Network Cost and Network Travel Cost Reliability}

This example considers the same scenario as in the previous example but focuses on demonstrating the effect of demon coalitions to the demons' payoffs (i.e., total network expected cost) and network travel cost reliability. The tolerance used in reliability is equal to 1.2 times the user equilibrium's total network cost as in Cassir et al. (2003). The minimum payoff of each demon under each case is shown in Table 2. This table clearly shows that the minimum payoff increases with a decreasing number of coalitions, as the extent of cooperation increases with a decreasing number of coalitions in our example. The payoff is increased marginally (2\%) when any two demons form one coalition and select links non-cooperatively against the third one. In the extreme case, where there is only one coalition, the payoff is increased by $20 \%$ when compared with the non-cooperative case. In terms of network travel cost reliability, the reliability decreases from 0.11 to 0 when the number of coalitions increases from 1 to 3 .

Table 2

The effect of the extent of cooperation to the demons' payoff and network travel cost reliability

\begin{tabular}{|c|c|c|}
\hline Number of coalitions & Minimum payoff of each demon & Reliability \\
\hline 3 & $\$ 35797$ & 0.11 \\
\hline 2 & $\$ 36675$ & 0 \\
\hline 1 & $\$ 43280$ & 0 \\
\hline
\end{tabular}

The three cases result in different link selection strategies. When all demons are non-cooperative, each demon selects a link for damage with a probability of 1/3. However, when all demons cooperate together, all demons will cooperatively select different links for damage with a probability of 1 so as to maximize the payoff. It is because the capacity of a link will not be reduced further if one additional demon selects the same link for damage. Therefore, the best strategy is not to let more than one demon to select a link for damage. When there are two demons co- 
operating against the third one, the third one selects each link for damage with a probability of $1 / 3$ and the demon coordinator selects each combination of two different links for damage with a probability of $1 / 3$. This means there are at least two links selected for damage but all links may not be selected for damage simultaneously. Hence, the payoff of each demon is lower than that of the single coalition case because of the possibility of two demons selecting the same link for damage. One interesting observation is that even if two demons form one coalition against the third demon, the payoffs of all three demons are higher than their payoff when no demon works together. That is, the third demon also receives some benefit resulting from the cooperation between the other two demons.

If the free-flow travel cost of one link were increased, the minimum payoff also increased with a decreasing number of coalitions. This shows that more cooperation will increase the minimum payoff of each demon and the total expected network cost regardless of the geometry of the network.

This example implies that the multi-demon approach (that assumes no cooperation between demons) can underestimate the worst case's total expected network cost and overestimate the worst case's the network reliability when the Stackelberg-Nash solution is not obtained. One should employ the proposed StackelbergNash formulation to determine the worst case's total expected network cost and reliability.

\subsection{Stochastic Braess' Paradox and Travel Cost Reliability Paradox}

This example demonstrates the existence of stochastic Braess' paradox and a travel cost reliability paradox in the proposed Stackelberg-Nash problem, the proposed partial-cooperative Nash problem and the classical pure-Nash game problem. The example network together with its link cost functions are shown in Figure 1. This network is similar to the classical Braess network but all free-flow travel costs are positive and the performance functions are nonlinear. For illustrative purposes, the number of users and demons are respectively set to equal 1000 and 1, although other numbers can be used to illustrate the existence of the paradoxes. Like previous example, the capacity of each link is assumed to be reduced by half if the demon selects the link for damage, and the tolerance used is equal to 1.2 times the user equilibrium's total network cost.

Under the above setting, the two proposed problems give the same solution as the classical pure Nash game problem. Before link addition, both routes carry equal flows, and the demon selects links 3 and 4 for damage with the corresponding link selection (or scenario) probabilities $q_{3} \geq 0$ and $q_{4}=1-q_{3} \geq 0$. The resultant total expected network cost (TENC) is $\$ 66500$ and the network (or OD) travel cost reliability is 1 .

After link addition, all the users use the new route, 1-3-4-2 although the demon selects the same two links for damage with the same probabilities as before. Consequently, the resultant $T E N C$ decreases to $\$ 76000$ (or by 14\%) and the stochastic 
Braess paradox occurs. In terms of network (or OD) travel cost reliability, it drops to 0 and the reliability paradox occurs.

The main reason of the occurrence of the two paradoxes is that the users are non-cooperative between themselves. Therefore, the overall system performance in terms of either reliability or expected cost is not necessarily improved when the capacity of the network is improved, or vice versa.

In fact, the existence of these paradoxes depends on many factors. Like the classical Braess paradox, their occurrence depends on the geometry, the functional form of link cost function, and the number of users (i.e., demand level). Unlike the classical Braess paradox, their occurrence also depends on the number of demons and the extent of cooperation. In particular, the occurrence of the reliability paradox also depends on the tolerance used in defining the reliability. Due to space limitation, the examples are not shown here.

The implication of this example is that the TENC and network travel cost reliability should be considered in network design to avoid the occurrence of the stochastic Braess and reliability paradoxes. Otherwise, after network expansion, the network performance may be worse off in terms of TENC, and the network can be less reliable in terms of network travel cost reliability.

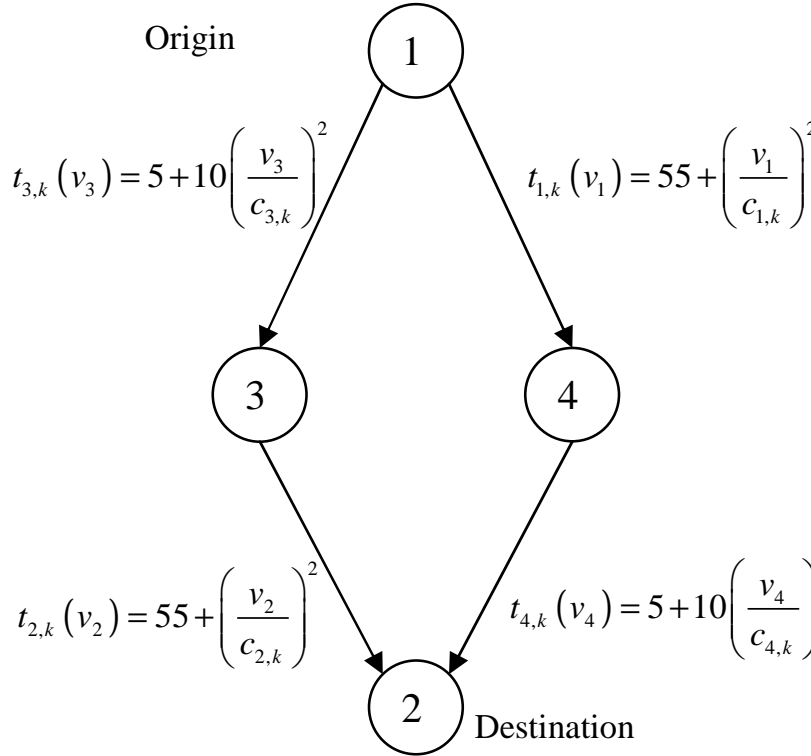

(a) Braess' network before im-

provement

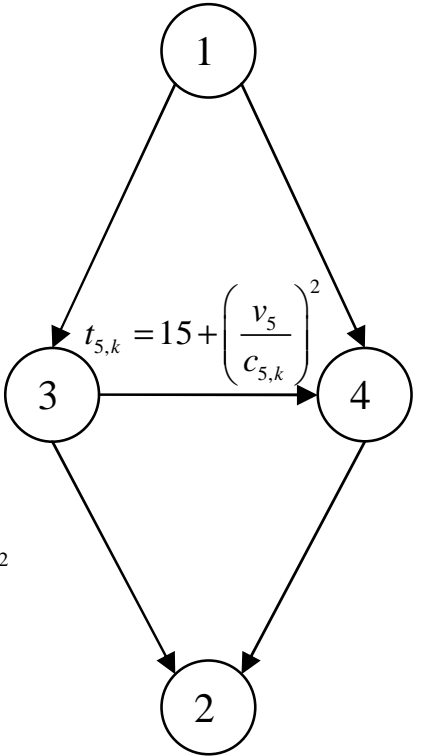

(b) Braess' network after improvement

Fig. 1. Braess' network. 


\section{Conclusions}

This paper develops two game-theoretic formulations based on cooperative game theory. The properties of the formulations are examined. This paper also discusses under what conditions, the multi-demon formulation can produce the same solution as the two proposed formulations. The relationships between the three formulations are examined. Numerical examples are provided to study the effects of the level of cooperation on total network expected cost and travel cost reliability and illustrate two paradoxical phenomena in terms of expected network travel cost and network travel cost reliability. Further insights into the implications for reliable network design are provided if these paradoxes are ignored and/or classical game-theoretic methods are adopted. As a remark, this paper suggests to adopt the Stackelberg-Nash approach to measuring the worst-case network reliability as it takes the user behavior into account when determining the worst-case network performance.

This paper opens up many future research directions. First, the very important question of how many demons are appropriate to assume remains unanswered. Extensive studies on real networks involving data on previous incidents are required to rectify this. Second, it is not clear whether the occurrence of stochastic Braess' paradox implies the occurrence of reliability paradox and vice versa. This is left to future studies. Finally, efficient global optimization methods for the proposed formulations have not been developed yet. Developing them is one of the important and challenging future research directions.

\section{References}

Asakura, Y., Kashiwadani, M., 1991. Road network reliability caused by daily fluctuation of traffic flow. Proceedings of the $19^{\text {th }}$ PTRC Summer Annual Meeting, Brighton, U.K., Sep 1991, pp. 73-84.

Al-Deek, H., Emam, E.B., 2006. New methodology for estimating reliability in transportation networks with degraded link capacities. Journal of Intelligent Transportation Systems 10(3), 117-129.

Bell, M.G.H., 2000. A game theory approach to measuring the performance reliability of transport networks. Transportation Research Part B 34(6), 533-545.

Bell, M.G.H., Cassir, C., 2002. Risk-averse user equilibrium traffic assignment: an application of game theory. Transportation Research Part B 36(8), 671-681.

Bell, M.G.H., Schmöcker, J.D., 2002. Public transport network reliability: Topological effects. Proceedings of the 3rd International Conference on Traffic and Transportation Studies (ICTTS), Guilin, China, July 23-25, 2002, pp. 453-460.

Braess, D., 1968. Uber ein paradoxon der verkehrsplanung. Unternehmenforschung 12, 258-268.

Cassir, C., Bell, M.G.H., Schmöcker, J.-D, 2003. A normative assessment of transport network reliability based on game theory. In: Bell, M.G.H., Iida, Y. (Eds.), The Network Reliability of Transport, Pergamon, UK, pp. 225-243.

Chen, A., Ji, Z.W., Recker, W., 2002. Travel time reliability with risk-sensitive travelers. Transportation Research Record 1783, 27-33. 
Chen, A., Kim, J., Zhou, Z., Chootinan, P., 2007. Alpha reliable network design problem. Transportation Research Record 2029, 49-57.

Chen, A., Yang, H., Lo, H.K., Tang, W.H., 1999. A capacity related reliability for transportation networks. Journal of Advanced Transportation 33(2), 183-200.

Chen, A., Yang, H., Lo, H.K., Tang, W.H., 2002. Capacity reliability of a road network: an assessment methodology and numerical results. Transportation Research Part B 36(3), 225252.

Higatani, A., Kitazawa, T., Tanabe, J., Suga, Y., Sekhar, R., Asakura,Y., 2009. Empirical Analysis of Travel Time Reliability Measures in Hanshin Expressway Network. Journal of Intelligent Transportation Systems 13(1), 28-38.

Heydecker, B.G., Lam, W.H.K., Zhang, N., 2007. Use of travel demand satisfaction to assess road network reliability. Transportmetrica 3(2), 139-171.

Iida, Y. and Wakayabashi, H. (1989) An approximation method of terminal reliability of road network using partial path and cut set. Proceedings of the 5th World Conference, Vol. IV, Yokohama, Japan, 367-380.

Kaparias, I., Bell, M.G.H., Belzner, H., 2008. A new measure of travel time reliability for invehicle navigation systems. Journal of Intelligent Transportation Systems 12(4), 202-211.

Lam, W.H.K., Shao, H., Sumalee, A., 2008. Modeling impacts of adverse weather conditions on a road network with uncertainties in demand and supply. Transportation Research Part B 42(10), 890-910.

Lo, H.K., 2002. Trip travel time reliability in degradable transport networks. In: Taylor, M. (Eds.), Transportation and Traffic Theory in the $21^{\text {st }}$ Century, Pergamon, UK, pp. 541-560.

Lo, H.K., Luo, X.W., Siu, B.W.Y., 2006. Degradable transport network: Travel time budget of travelers with heterogeneous risk aversion. Transportation Research Part B 40(9), 792-806

Lo, H.K., Tung. Y.K., 2003. Network with degradable links: capacity analysis and design. Transportation Research Part B 37(4), 345-363.

Nie, Y., Fan, Y.Y., 2006. Arriving-on-time problem - Discrete algorithm that ensures convergence. Transportation Research Record 1964, 193-200.

Siu, B.W.Y., Lo, H.K., 2008. Doubly uncertain transportation network: Degradable capacity and stochastic demand. European Journal of Operational Research 191(1), 166-181.

Shao, H., Lam, W.H.K., Tam, M.L., Yuan, X.M., 2008. Modelling rain effects on risk-taking behaviours of multi-user classes in road networks with uncertainty. Journal of Advanced Transportation 42(3), 265-290.

Sumalee, A., Watling, D.P., Nakayama, S., 2006. Reliable network design problem - Case with uncertain demand and total travel time reliability. Transportation Research Record 1964, 81 90.

Sumalee, A., Watling, D.P., 2008. Partition-based algorithm for estimating transportation network reliability with dependent link failures. Journal of Advanced Transportation 42(3), 213238.

Szeto, W.Y., O'Brien, L., O'Mahony, M., 2006. Risk-averse traffic assignment with elastic demands: NCP formulation and solution method for assessing performance reliability. Network and Spatial Economics 6(3), 313-332.

Szeto, W.Y., O'Brien, L., O'Mahony, M., 2007. Generalisation of risk averse traffic assignment. In: Allsop, R.E., Bell, M.G.H., Heydecker, B.G. (Eds.), Transportation and Traffic Theory, Elsevier Science, Amsterdam, pp. 127-153.

Taylor, M.A.P., 1999. Dense network traffic models, travel time reliability and traffic management. I: General introduction. Journal of Advanced Transportation 33(2), 218-233.

Yang, H., Lo, H.K., Tang, W.H., 2000. Travel time versus capacity reliability of a road network. In: Bell, M.G.H., and Cassir, C. (Eds.), Reliability of Transport Networks, Research Studies Press, Baldock, pp. 191-138. 